\title{
Les médicaments: reflet de la problématique du progrès médical
}

\section{R. Bruppacher ${ }^{1}$}

Pendant des millénaires les hommes avaient confiance dans les vertus guérissantes de médicaments. Jetant un regard en arrière nous constatons que, jusque dans un passé récent, cette confiance a, dans la plupart des cas, contribué davantage à la guérison que le médicament lui-même. Au cours des quarante dernières années la pharmacothérapie a pris un essor fulgurant. Nous disposons aujourd'hui d'une multitude de médicaments dont l'efficacité a été minutieusement établie. Ils sont soumis à un contrôle permanent quant à leur qualité et leur sécurité, contrôle qui surpasse de loin la mesure habituelle.

En dépit de ce fait, la confiance dans le profit du recours à la pharmacothérapie a fait place, dans de larges milieux, pour une part tout au moins, à un malaise et à une méfiance. Parallèlement à l'augmentation de l'efficacité, l'on assiste à une augmentation des dangers que présente une utilisation mal comprise. Comme cela se présente régulièrement lorsque les potentialités de la science moderne vont de pair avec la possibilité d'une défaillance humaine, un sentiment de menace surgit. Le médicament est très souvent au centre des critiques formulées à l'égard de la santé publique. Cette dernière est souvent ressentie comme une intervention douloureuse et injustifiée par de nombreuses personnes vouant toutes leurs forces et leur intelligence à la lutte contre la souffrance humaine. Ces critiques ne s'adressent cependant pas aux défenseurs du progrès médical, mais aux effets secondaires non voulus. La tragédie de la thalidomide a laissé une impression mémorable. Conformément au principe directeur «Primum nil nocere», des contrôles par l'Etat ont été institués, soumettant le développement, la diffusion et l'utilisation de médicaments à des contraintes sans cesse croissantes. Il en résulte que seuls des groupes de travail hautement spécialisés conservent la possibilité de développer, fabriquer et mettre en vente avec quelques chances de succès des préparations pharmaceutiques. L'industrie pharmaceutique s'est ainsi élevée au rang de partenaire important pour les professionnels de la médecine.

Cependant, les progrès réalisés dans le domaine de la médecine ont été liés à une augmentation massive du coût pour la santé publique. Quand bien même les médicaments participent à cette augmentation à un degré beaucoup plus modeste que d'autres éléments du traitement et des soins, les efforts visant une diminution des coûts sont facilement entrepris dans cette direction, non liée directement à la prise en charge personnelle, mais justifiée plus précisément par des considérations d'ordre industriel. La mise à la charge des assurances sociales, subventionnées par l'Etat, des coûts du traitement médical, a eu pour conséquence que d'autres instances se sont intéressées au contrôle de l'utilisation des médicaments.

Des questions touchant au traitement médical sont discutées, d'une manière croissante, par le public. Des informations et des «instructions» concernant l'utilisation de préparations, même soumises à ordonnance, sont également publiées par des milieux non médicaux dans la presse s'adressant à un public non initié. Le rôle traditionnel des professionnels de la santé est mis en question.

La Société Suisse de Médecine sociale et préventive, dans ses efforts visant à offrir un forum pour des discussions interdisciplinaires servant de point de départ à des solutions objectives tenant compte de l'intérêt de notre population, a placé ses journées d'automne, consacrées traditionnellement à un thème d'actualité du domaine de la santé publique, sous le titre «Le médicament dans le système de santé publique d'aujourd'hui». Les exposés et les rapports publiés dans le présent numéro donnent un reflet vivant des débats animés mais menés dans un esprit constructif auxquels cette matière, sujette à conflits, a donné lieu. En toute logique, c'est la Société suisse des pharmaciens et plus particulièrement l'Ordine dei Farmacisti del Cantone Ticino, dont le président, M. Luigi Varini, a fonctionné en qualité de président du Comité d'organisation, qui a été choisie comme partenaire pour cette manifestation. Nous exprimons à M. Varini et également au professeur Boris Luban-Plozza, président de ces journées, ainsi qu'à tous les participants nos remerciements les plus chaleureux pour les efforts qu'ils ont accomplis au profit des hommes vivant dans la souffrance pour rapprocher les positions divergentes et pour désamorcer les problèmes touchant au recours aux médicaments.

1 Privat-docent, Dr méd., Dr P. H., Abteilung für Sozial- und Präventivmedizin der Universität Basel, c/o Programmleitung NFP 8, Kantonsspital, CH-4031 Basel. 